\title{
Processo de cuidado para prevenção de quedas em idosos: teoria de intervenção práxica da enfermagem
}

Care process for fall prevention in the elderly: theory of nursing praxis intervention

Proceso de atención para la prevención de caídas en ancianos: teoría de la intervención práxica de la enfermería

\section{Larissa Padoin Lopes ${ }^{1}$ (1) lara Sescon Nogueira ${ }^{1}$ (D) Jhenicy Rubira Dias ${ }^{1}$ (c)}

Vanessa Denardi Antoniassi Baldissera ${ }^{1}$ (1)

1. Universidade Estadual de Maringá.

Maringá, PR, Brasil.
Autor correspondente:

Larissa Padoin Lopes.

E-mail: laripadoinlopes@gmail.com

Recebido em 01/07/2021.

Aprovado em 06/12/2021.

\section{RESUMO}

Objetivo: analisar o processo de cuidado para prevenção de quedas em idosos na perspectiva teórica e metodológica da Teoria de Intervenção Práxica da Enfermagem em Saúde Coletiva. Métodos: estudo transversal descritivo de abordagem qualitativa do tipo pesquisa-cuidado, realizado com participantes de um grupo de convivência de uma Unidade Básica de Saúde em MaringáPR. Os dados foram coletados a partir de entrevistas semiestruturadas e analisados utilizando a Teoria de Intervenção Práxica da Enfermagem em Saúde Coletiva. Resultados: realizou-se a captação e interpretação dos dados nas dimensões estrutural particular e singular, seguido da avaliação de saúde e identificação dos principais riscos intrínsecos e extrínsecos para quedas dos idosos. Os principais diagnósticos e intervenções de enfermagem foram elencados segundo a CIPE ${ }^{\circledR} / \mathrm{CIPESC}^{\circledR}$. Conclusões e implicações para prática: foi possível analisar o processo de cuidado na prevenção de quedas junto aos idosos por meio da perspectiva teórica e metodológica na Teoria de Intervenção Práxica da Enfermagem em Saúde Coletiva, sendo proposto um plano de intervenções de enfermagem.

Palavras-chave: Acidentes por Quedas; Cuidados de Enfermagem; Idoso; Prevenção de Acidentes; Teoria de Enfermagem.

\section{ABSTRACT}

Objective: to analyze the care process for the prevention of falls in the elderly from the theoretical and methodological perspective of the Theory of Nursing Praxis Intervention in Collective Health. Methods: this is a descriptive cross-sectiona study with a qualitative approach and care-research method carried out with participants of a community group of a Basic Health Unit in Maringá (Paraná State). Data were collected through semi-structured interviews and analyzed using the Theory of Nursing Praxis Intervention in Collective Health. Results: data were acquired and interpreted in the structural, particular and singular dimensions, and consequently, the health assessment was carried out, and the main intrinsic and extrinsic risks for falls among the elderly were identified. The main nursing diagnoses and interventions were listed according to the ICNP/ CIPESC. Conclusion and implications for practice: it was possible to analyze the care process in preventing falls with the elderly through the theoretical and methodological perspective of the Theory of Nursing Praxis Intervention in Collective Health and propose a nursing intervention plan.

Keywords: Accidental Falls; Nursing Care; Aged; Accident Prevention; Nursing Theory.

\section{RESUMEN}

Objetivo: analizar el proceso de cuidado para la prevención de caídas en ancianos desde la perspectiva teórica y metodológica de la Teoría de la Intervención Práxica de la Enfermería en la Salud Colectiva. Métodos: estudio transversal descriptivo de enfoque cualitativo del tipo investigación-cuidado, realizado con participantes de un grupo de convivencia de una Unidad Básica de Salud en Maringá (estado brasileño de Paraná). Los datos fueron recogidos a partir de entrevistas semiestructuradas y analizados utilizando la Teoría de Intervención Práxica de la Enfermería en Salud Colectiva. Resultados: se realizó la captación e interpretación de los datos en las dimensiones estructural, particular y singular, seguido de la evaluación de salud e identificación de los principales riesgos intrínsecos y extrínsecos para caídas de ancianos. Los principales diagnósticos e intervenciones de enfermería fueron enumerados según la CIPE/CIPESC. Conclusión e implicaciones para la práctica: fue posible analizar e proceso de cuidado en la prevención de caídas junto a los ancianos por medio de la perspectiva teórica y metodológica de la Teoría de la Intervención Práxica de la Enfermería en Salud Colectiva, con una propuesta de un plan de intervenciones de enfermería. Palabras claves: Accidentes por Caídas; Atención de Enfermería; Anciano; Prevención de Accidentes; Teoría de Enfermería 


\section{INTRODUÇÃO}

No mundo, e também no Brasil, o número de idosos vem crescendo em ritmo acelerado, representando 12,5\% da população total brasileira, sendo que até ao ano de 2050 poderá atingir o incrível percentual de $30 \% .{ }^{1}$ Tal realidade implica em mudanças no perfil epidemiológico, contribuindo também para o aumento de agravos à saúde da pessoa idosa, sendo a queda, uma das principais ameaças à qualidade de vida do idoso. ${ }^{1}$ Sabe-se que os acidentes por quedas atingem cerca de $32 \%$ dos idosos com faixa etária de 65 a 74 anos e $51 \%$ dos idosos com mais de 85 anos, sendo que no Brasil, a prevalência de quedas em idosos que vivem na comunidade é de $27,6 \%$. $^{2,3}$

A literatura aponta que as quedas são resultado de uma interação entre os fatores intrínsecos (relacionados ao próprio indivíduo) e extrínsecos (relacionado ao meio ambiente). Os principais fatores de risco determinantes para quedas relacionam-se com a autoavaliação negativa da saúde, baixa acuidade visual, uso de polifarmácia, presença de doenças crônicas, comprometimento para a execução das atividades da vida diária, depressão, idade igual ou superior a 80 anos, e déficit cognitivo. ${ }^{2,4}$

Apesar dos acidentes por quedas em idosos serem considerados um problema de saúde pública, é passível de prevenção, sendo necessário e oportuno o rastreio do risco de quedas em idosos e a avaliação de saúde dos mesmos. ${ }^{5} \mathrm{Nessa}$ direção, ressalta-se a importante atuação dos profissionais enfermeiros que integram as equipes de Estratégia Saúde da Família (ESF) na Atenção Primária à Saúde (APS), pois buscam identificar o risco de quedas em idosos, desenvolver e implantar práticas de cuidados com foco na prevenção das quedas de modo integral. ${ }^{6}$

Assim, entre as ações de saúde desenvolvidas na APS, os grupos de convivência para idosos emergem enquanto cenário de promoção da saúde e prevenção de doenças e agravos, como é o caso das quedas em idosos. Na perspectiva da saúde do idoso, grupos de convivência da terceira idade potencializam a convivência social, a manutenção da autonomia e estimulam a aprendizagem coletiva de temáticas relacionadas à saúde. Diante disso, torna-se extremamente relevante promover ações de promoção de saúde e de prevenção de acidentes por quedas em idosos em grupos de convivência da terceira idade. ${ }^{7}$

Nesse contexto, e considerando que o número de quedas na população idosa vem crescendo progressivamente, é essencial identificar e avaliar o risco de quedas em idosos frequentadores de um grupo de convivência para a implementação de estratégias visando a prevenção da mesma. ${ }^{6}$ Apesar da literatura demonstrar pesquisas que avaliam o risco de quedas nos diversos contextos de atenção à saúde, há uma lacuna no que diz respeito aos estudos desenvolvidos com idosos frequentadores de grupos de convivência na APS. ${ }^{8}$

A partir dessa perspectiva, podemos destacar algumas teorias de enfermagem, dentre elas, a Teoria de Intervenção Práxica da Enfermagem em Saúde Coletiva (TIPESC), que compreende as contradições da realidade objetiva da
Enfermagem em Saúde Coletiva, tendo como base filosófica o materialismo histórico-dialético. A TIPESC busca a intervenção de Enfermagem por meio de uma metodologia dinâmica, dialetizada e participativa, por meio da captação e interpretação de um determinado fenômeno relacionado à saúde e doença de uma dada coletividade, a intervenção nessa realidade propriamente dita e a reinterpretação da realidade para avaliar se a mesma de fato resultou em melhorias ao alcance coletivo, ${ }^{9}$ coerente com o desenvolvimento de intervenções de enfermagem com foco na prevenção de quedas em idosos.

Desse modo, em parceria com uma equipe de saúde da APS, pesquisadoras buscaram o desenvolvimento de intervenções de enfermagem voltadas para os idosos frequentadores de um grupo de convivência vinculados a uma Unidade Básica de Saúde (UBS), e para isso, desenvolveram a presente pesquisa, colaborando com as práticas preventivas de quedas ao permitir identificar e avaliar o risco de quedas em idosos, visto que essa colaboração prescindiu do identificação prévia do risco de quedas e de saúde dos mesmos. Diante do exposto, o presente estudo objetivou analisar o processo de cuidado para prevenção de quedas em idosos na perspectiva teórica e metodológica da Teoria de Intervenção Práxica da Enfermagem em Saúde Coletiva.

\section{MÉTODO}

Tratou-se de um estudo qualitativo e delineado pela abordagem de Pesquisa-Cuidado (PC), que almejou identificar os problemas, os diagnósticos de enfermagem e propor intervenções de enfermagem para implementação e transformação dos cuidados de enfermagem no que se refere à prevenção de quedas em idosos. $^{10}$

A pesquisa foi realizada no período de março a agosto de 2020 durante o período de pandemia da COVID-19 em uma UBS, localizada no município de Maringá, situado na região Norte Central do estado do Paraná-Brasil, tendo como público-alvo 10 idosas frequentadoras de um grupo de convivência vinculado à referida UBS, intitulado "De bem com a vida". O público-alvo foi selecionado intencionalmente, por considerar o vínculo e oportunidade existente para um estudo dessa natureza.

Os critérios de inclusão para participação na pesquisa foram: 1) Ser participante do grupo denominado "De bem com a vida" independentemente do tempo em que participa do mesmo e; 2) Possuir idade igual ou superior a 60 anos. Como critérios de exclusão elegeu-se: 1) Não apresentar capacidade cognitiva preservada de acordo com o Mini Exame do Estado Mental (MEEM);2) Não ser localizada no domicílio ou não ser localizada após cinco tentativas de contato telefônico, no momento da coleta de dados. ${ }^{11}$ Durante uma reunião do grupo de convivência no mês de fevereiro de 2020, todos os integrantes foram convidados a participar do estudo. Seguindo os critérios, uma idosa não foi localizada, sendo excluída da pesquisa, totalizando a participação de nove idosas.

A coleta de dados foi realizada por acadêmicos de enfermagem capacitados, por meio de entrevistas individuais semiestruturadas, efetivadas inicialmente com três idosas a partir 
de visita domiciliar, e após o início da pandemia de COVID-19 no Brasil, por meio de contato telefônico com outras seis idosas. As entrevistas tiveram duração média de 34,49 minutos e foram audiogravadas, utilizando inicialmente um gravador de telefone celular, por ocasião da coleta ocorrer via visita domiciliar, sendo posteriormente utilizado o aplicativo gravador de chamada telefônica intitulado "Gravador de Chamadas - Cube ACR", para as coletas de dados via remota.

Os dados foram transcritos na íntegra por uma das pesquisadoras, organizados em planilha eletrônica do software Microsoft Excel $₫$ 2013 e analisados utilizando a TIPESC que compreende cinco etapas em seu processo, sendo elas: 1) Captação da realidade objetiva; 2) Interpretação da realidade objetiva; 3) Proposta de intervenção na realidade objetiva; 4) Intervenção na realidade objetiva e; 5) Reinterpretação da realidade objetiva. ${ }^{9}$ No presente estudo abordamos apenas as três primeiras etapas da TIPESC

Na primeira etapa - captação da realidade objetiva buscou-se compreender o fenômeno das quedas, descrever sua situcionalidade e historicidade. Para isso, os dados foram coletados utilizando os seguintes instrumentos: 1) Questionário para Avaliação do Risco de Quedas em Idosos, que é relacionado com a funcionalidade, incapacidade e saúde do idoso; 2) MEEM, para avaliação da função cognitiva, adaptado para aplicação por via telefônica, sendo excluído o tópico de linguagem e readequado o score de acordo com a seguinte pontuação: <16 pontos para altamente escolarizados, $<12$ pontos para fundamenta incompleto, e $<9$ pontos para analfabetos; 3) Índice de Katz; 4) Escala de Lawton e Brody; 5) Escala Ambiental do Risco de Quedas e; 6) Índice de Funcionalidade Clínico Funcional-20 (IVCF20), considerando a importância da avaliação da capacidade funcional e da vulnerabilidade do idoso no rastreio do risco de quedas em idosos. , $^{, 11-14}$

Para além dos instrumentos mencionados, também foi utilizado um questionário para caracterização sociodemográfica e de saúde das idosas, elaborado pelos próprios pesquisadores composto por questões referentes à idade, sexo, escolaridade, estado civil, ocupação, presença de morbidades e doenças crônicas, polifarmácia, utilização de dispositivo para auxílio de mobilidade, realização de atividade física e social, e se recebeu alguma orientação de profissional de saúde sobre prevenção e risco de quedas.

A segunda etapa compreendeu a interpretação da realidade objetiva, em que se buscou uma aproximação das teorias explicativas da ocorrência dos fenômenos das quedas, buscando analisar as contradições dialéticas. Dessa forma, a interpretação da realidade objetiva ocorreu a partir da avaliação de saúde e dos riscos intrínsecos e extrínsecos para quedas analisando os dados obtidos. Foram elencados os principais diagnósticos de enfermagem no que se refere às quedas segundo a Classificação Internacional para a Prática de Enfermagem (CIPE) e a Classificação Internacional das Práticas de Enfermagem em Saúde Coletiva (CIPESC ${ }^{\circledR}$ ), que estão ancoradas na Teoria das Necessidades Humanas Básicas de Wanda de Aguiar Horta.
Na terceira e última etapa, a de proposta de intervenção na realidade objetiva, buscou-se a criação de um plano de intervenções de enfermagem focando nas principais vulnerabilidades relacionados ao risco de quedas, preconizado conforme as intervenções de enfermagem segundo a CIPE ${ }^{\circledR} / \mathrm{CIPESC}^{\circledR}$, elaborado a partir dos diagnósticos identificados na etapa anterior. ${ }^{9}$

A pesquisa respeitou os preceitos éticos orientados pela Resolução no 466/2012 e no 510/2016 do Conselho Nacional de Saúde. A pesquisa faz parte de um estudo mais abrangente e possui aprovação do Comitê Permanente de Ética em Pesquisas com Seres Humanos, sob parecer $n$ ํㅜ 1.954.350/2017 (CAEE: 37457414.6.0000.0104) aprovado em março de 2017.

\section{RESULTADOS E DISCUSSÃO}

\section{Captação e interpretação da realidade objetiva}

A captação da realidade objetiva ocorreu a partir da coleta de dados junto as idosas e permitiu compreender as condições de saúde das mesmas e os riscos para quedas, juntamente com a identificação dos diagnósticos de enfermagem segundo a CIPE $^{\circledR}$ e a CIPESC $^{\circledR}$. Dessa forma, elucidou a dimensão estrutural, por meio da compreensão da estrutura de saúde local; singular, a partir da caracterização sociodemográfica e de saúde e; representando a dimensão particular, os saberes e as orientações sobre a prevenção de quedas. Tais dimensões estão apresentadas e detalhadas a seguir:

\section{Dimensão estrutural}

Para a categoria estrutural, observou-se o cenário da referida UBS, localizada no município de Maringá-PR, integrado à Rede de Atenção à Saúde do Idoso. Sob o ponto de vista organizacional, o sistema de saúde para o atendimento qualificado do idoso, no estado do Paraná, é norteado pela Rede de Atenção à Saúde do Idoso e Linha Guia da Saúde do Idoso. ${ }^{14}$

A realidade é que as quedas, na população idosa, aumentam conforme o avanço da idade do idoso e conforme o grau de fragilidade e, assim, para o controle desse evento, pelo menos uma vez anualmente deve-se investigar a ocorrência de quedas e a marcha, coerente com os cuidados praticados pelo enfermeiro integrante da equipe de ESF da UBS investigada. Para além disso, quando os idosos relatam não ter sofrido quedas prévias, a linha guia de saúde orienta que a APS desenvolva práticas educativas com foco na prevenção das quedas e nos principais fatores de risco. ${ }^{14}$

No município em que se realizou a pesquisa existem 35 UBS. ${ }^{15}$ Para além disso, sabe-se que o município possui uma taxa de crescimento da população idosa de $4 \%$ ao ano, e as quedas nos idosos são um dos principais agravos que aumentam juntamente com a elevação dessa população, sendo necessário a sensibilização das UBS quanto à prevenção de quedas em idosos. ${ }^{16}$

Com relação à UBS cenário dessa pesquisa, foi inaugurada em 2013 e atualmente atende cerca de oito mil pessoas, possui uma equipe de ESF e dois profissionais que faziam parte do 
extinto Núcleo Ampliado de Saúde da Família (NASF). Além disso, dentro da área de abrangência da UBS fazem parte instituições filantrópicas de apoio ou permanência de idosos. Dentre os serviços ofertados pela UBS para o público idoso, destaca-se o grupo de convivência "De bem com a vida", que ocorre semanalmente, por meio de atividades de lazer social juntamente com atividades de Educação em Saúde, coordenado e executado por profissionais do serviço em parceria com uma instituição de ensino superior pública.

\section{Dimensão particular}

No tocante da dimensão particular, foram avaliados os aspectos sociodemográficos e de saúde das idosas, bem como a participação delas no grupo de convivência e as suas vivências com relação às atividades educativas que tiveram no grupo sobre prevenção de quedas.

Todas as idosas entrevistadas participam do grupo há pelo menos um ano, e relataram que começaram a participar do grupo por convite de alguém da equipe de saúde ou de vizinhas, demonstrando gostar da participação do grupo por oferecem um momento de aprendizagem e de criação de vínculo, tanto com a equipe de saúde quanto com outras idosas frequentadoras do grupo. Duas idosas participam de outros grupos sociais além do grupo de convivência "De bem com a vida", sendo citado os grupos sociais de igrejas. A constância da participação das idosas no grupo é considerado um fator importante em meio a temática, já que é uma ferramenta que possibilita o aumento da qualidade de vida dos idosos, uma vez que eles transformam e constroem saberes, a partir da participação em atividades educativas. ${ }^{17}$

No que diz a respeito à caracterização sociodemográfica dos participantes do grupo de convivência, todos os nove idosos eram do sexo feminino, com idades de 61 a 86 anos, média de 74,66 anos. Estudos na literatura indicam que o sexo feminino e o avançar da idade são fatores de aumento para o risco de quedas. ${ }^{18,19}$

Em relação ao estado civil, uma idosa era divorciada, quatro eram casadas e quatro eram viúvas. Sobre a escolaridade, quatro idosas não eram alfabetizadas e cinco possuíam o ensino fundamental incompleto, cursando até a $4^{\circ}$ série. Referente à ocupação delas, cinco eram aposentadas, três eram do lar e uma estava empregada. Sobre a religião, quatro eram católicas, três eram evangélicas e duas adventistas.

A prevalência de idosas na pesquisa com baixa escolaridade pode ser considerado um fator de risco para quedas, e de acordo com evidências encontradas na literatura, demonstram que a baixa escolaridade influencia de forma negativa na funcionalidade dos idosos, assim como a ocupação aposentada(o) e o estado civil viúva(o) também estão associados ao risco de quedas, visto que esses fatores podem ser considerados limitantes e incapacitantes para a vida do idoso. ${ }^{18-20}$

No que se refere à autopercepção de saúde, cinco idosas possuíam autoavaliação de saúde negativa, considerando sua saúde como "regular", e quatro idosas avaliaram a saúde positivamente, sendo que uma considerou sua saúde como "muito boa" e outras três como "boa". Uma idosa referiu possuir cuidador, sendo a filha declarada como cuidadora principal. Oito idosas relataram ter alguma doença crônica (depressão, hipotireoidismo, hipercolesterolemia, hipertensão arterial sistêmica, diabetes, osteoporose, catarata, ansiedade e/ou problemas respiratórios). Sobre a utilização de medicamentos de uso contínuo, oito idosas afirmaram que fazem uso, e duas utilizam polifarmácia. Dentre os medicamentos mais citados estão a levotiroxina, sinvastatina, clonazepam, losartana e besilato de anlodipino.

Considerando que mais da metade das idosas investigadas possuem autoavaliação negativa de saúde, estudos demonstram a associação da autopercepção negativa da saúde com uma maior chance de quedas. A autopercepção negativa, por sua vez, está associada com indicadores sociodemográficos, condições de saúde, diminuição da funcionalidade, prevalência de doenças crônicas e o fato de não praticar atividade física. ${ }^{21,22}$

As patologias relacionadas com o sistema cardiovascular também são consideradas um fator importante e de risco para quedas. Idosos portadores de doenças cardiovasculares têm maiores chances de sofrerem quedas do que aqueles que não tem, uma vez que o uso de medicamentos anti-hipertensivos pode aumentar o risco de quedas, já que dentre os seus efeitos colaterais estão a hipotensão postural, tonturas e necessidade de urinar com maior frequência. ${ }^{23} \mathrm{Na}$ presente pesquisa, cinco idosas fazem o uso de anti-hipertensivos, sendo considerado um risco para quedas que necessita de atenção.

Em contrapartida, um estudo demonstrou alta prevalência do uso de psicofármacos nos idosos, representando $93 \%$ das prescrições, e que pacientes que fazem uso desses medicamentos apresentam sete vezes mais risco de quedas. ${ }^{24} \mathrm{Ainda}$, a literatura afirma que o uso por idosos de cinco ou mais drogas associadas podem elevar o risco de quedas, e um estudo demonstrou que a polifarmácia causou de 1,5 a 2 vezes mais probabilidade de um idoso cair. Sendo assim, a polifarmácia é um fator importante a se considerar para o risco de quedas, e nas participantes da presente pesquisa duas fazem o uso de polifarmácia. ${ }^{25}$

Para além disso, cinco idosas relataram visão prejudicada e quatro fazem uso de óculos. A literatura aponta a diminuição da acuidade visual como um dos principais fatores relacionados à queda no âmbito domiciliar, pois interfere na marcha, podendo potencializar o risco de quedas quando acompanhada por outras patologias sensoriais e alterações no equilíbrio. ${ }^{26}$

No tocante à capacidade funcional das idosas, cinco foram classificados como robustas e quatro em risco de fragilização, enfatizando que nenhuma das idosas do grupo foi classificada como frágil. Todas as nove idosas possuíam capacidade cognitiva preservada segundo o MEEM. Sobre a capacidade cognitiva, ressalta-se que idosos considerados frágeis estão mais susceptíveis a cair no domicílio quando realizam atividades básicas de vida diária, além de que idosos em risco de fragilização transitam para a fragilidade em um período curto de 12 meses, sendo que a fragilidade se torna um risco para que ocorra quedas recorrentes. ${ }^{27,28}$ 
Um estudo apontou que o risco de quedas é maior em idosos com déficit cognitivo e escore do MEEM entre 24 e 30 pontos, e isso ocorre devido a uma má percepção e avaliação dos perigos ambientais e das próprias habilidades que podem ocasionar quedas. Outro fator que também pode ocasionar quedas e está relacionado com a capacidade cognitiva do idoso, é a depressão, doença que altera o estado cognitivo, e por vezes há a necessidade de utilização de medicamentos cujos efeitos colaterais aumentam ainda mais o risco para quedas. ${ }^{2}$

Para a avaliação da capacidade funcional dos idosos no presente estudo foram utilizados dois instrumentos, o primeiro, o Índice de Katz, que avaliou as Atividades Básicas de Vida Diária (ABVD), e o segundo, a Escala de Lawton e Brody, que avaliou as Atividades Instrumentais da Vida Diária (AIVD). Conforme as pontuações, todas as idosas participantes da pesquisa foram classificadas como independentes nas duas escalas. A literatura evidencia que quanto maior o comprometimento do idoso em executar suas atividades rotineiras, e eventualmente dependência nas atividades de vida diária, maior imobilidade e consequente atrofia muscular, podendo resultar em novas ocorrências de quedas. ${ }^{18-22}$

No que se refere às orientações para a prevenção de quedas, duas idosas relataram não ter recebido orientação de um profissional de saúde sobre a temática. As idosas que receberam orientações elucidaram que foram do tipo palestra e que ocorreram no próprio grupo de convivência, sendo explanado sobre os fatores de risco para quedas e os cuidados preventivos, como explicitado nos discursos das idosas que se seguem:

Eu recebi lá no grupo mesmo [...] ter cuidado, não deixar tapete no meio da casa, não deixar chinelo no meio da casa (I1).

Pra pessoa andar, saber andar direito, e se escorregar ou tem que tratar direito [...] (I2).

[...]Que não pode usar o tapete [...] ter cuidado de preferência e andar com sapatinho fechado, não de chinelo (I3).

Essas orientações apreendidas pelas idosas corroboram com um estudo que traz que as principais estratégias preventivas de quedas de idosos são retirar tapetes e organizar os móveis no domicílio, além da revisão no uso de medicamentos, a utilização de óculos para correção da visão, entre outros. ${ }^{29}$

\section{Dimensão singular}

Sobre a avaliação ambiental do risco de quedas, seis idosas afirmaram ter áreas desimpedidas, com barras de apoio, revestimentos uniformes e tapetes bem fixos. No fator iluminação, nove idosas relataram iluminação suficiente para clarear toda a superfície de marcha no interior de cada cômodo, incluindo degraus, iluminação exterior suficiente para iluminar toda a entrada exterior e ter interruptores acessíveis na entrada dos cômodos. Seis idosas possuem sentinela iluminando o quarto, o corredor e o banheiro, e possuem cama com luz indireta.
As nove idosas possuem guarda-roupa com cabides facilmente acessíveis, cama com boa altura, e uma das idosas possuí cadeira no quarto que permite sentar-se para se vestir Quanto ao banheiro, nove idosas possuem lavabo facilmente acessível e bem fixo, cinco idosas possuem área do chuveiro antiderrapante, e sete com box de abertura fácil e cortina bem fixa. Oito idosas possuem armários acessíveis sem necessidade do uso de escada e três possuem pia sem vazamento e que permite a entrada de cadeira de rodas se necessário.

Móveis de difícil acesso, mal posicionados ou inacessíveis podem contribuir para o aumento do risco de quedas, uma vez que os idosos possuem hábitos perigosos como subir em bancos para alcançar os objetos, e para além disso, o local de maior prevalência de quedas é o banheiro devido os fatores de risco presentes que podem ocasionar as quedas. ${ }^{30,31}$ Todas as idosas do grupo de convivência investigadas não possuem como fator de risco ambiental para quedas a dificuldade de acesso aos móveis em seu domicílio.

Quatro idosas possuem escadas em suas residências, e dentre essas, uma não possuí revestimento antiderrapante com marcação do primeiro e último degrau com faixa amarela, e outra não possui uniformidade nos degraus, altura dos espelhos e profundidade dos degraus constantes. Duas idosas possuem corrimão bilateral, corrimão sólido e corrimão que se prolonga além do primeiro e último degrau, e nenhuma idosa possuí espelho do degrau fechado com lixas antiderrapantes. Os riscos de quedas relacionados com escadas e apresentados pelas idosas da pesquisa estão de acordo com outros estudos que evidenciam a escada como o ambiente menos adequado do domicílio, devido por exemplo, a ausência de piso antiderrapante ou corrimão bilateral. ${ }^{30,32}$

No rastreio dos riscos intrínsecos para quedas, ${ }^{5}$ quando investigado o que as idosas estiveram sentindo no último mês, duas relataram tontura quando em pé ou caminhando, duas sentiram fraqueza em todo corpo, uma apresentou fraqueza no corpo todo, duas com fraqueza só nas pernas, duas com dificuldades para caminhar, e duas com cansaço ou esgotamento. Estudo demonstrou que um dos fatores que levam idosos a cair foi sentir tontura frequente, apresentando a tontura como um fator para o risco de quedas, e como observado na presente pesquisa, sintomas de cansaço, esgotamento, fraqueza ou tontura foram frequentes. ${ }^{31}$

Com relação à força das idosas para executar as atividades de vida diária, seis afirmaram que diminuiu no último ano. Quatro idosas acreditam que o seu modo de caminhar está mais devagar no último ano quando comparado com o ano anterior, e nenhuma idosa utiliza algum tipo de apoio ou ajuda para caminhar. Sabese ainda que existe maior ocorrência de quedas em idosos que apresentam redução da força de preensão, redução da velocidade de marcha e exaustão. ${ }^{4}$

No que diz respeito a prática de exercício físico, cinco idosas afirmaram realizar alguma atividade física pelo menos duas vezes na semana por no mínimo 30 minutos. Quanto ao tempo que a idosa faz exercício regularmente, uma relatou fazer 
de um a dois anos, e outras quatro idosas praticam de dois a três anos, se tornando um achado interessante, uma vez que a atividade física contribui significativamente reduzindo o número de quedas em idosos. ${ }^{33}$ Das participantes, seis informaram que sua capacidade de fazer tarefas do dia a dia diminuiu comparado com o ano anterior.

No que concerne à perda de peso no último ano, duas idosas afirmaram que perderam peso e que foi mais de três quilos. O emagrecimento pode tornar-se um risco para quedas nessas idosas, já que a literatura aponta maior ocorrência de quedas em idosos com perda de peso, visto que a perda de peso é um indicativo de sarcopenia e um dos fatores que podem ocasionar fragilidade nos idosos e consequentemente aumentar o risco de quedas. ${ }^{4,34}$

Quando as idosas foram questionadas se já caíram no último ano, duas afirmaram que sim, e que caíram duas vezes. Dessas quedas, três ocorreram na rua e uma no próprio domicílio. Em relação a circunstância da queda, uma afirmou que estava em pé, desequilibrou e caiu, e outra afirmou que estava caminhando, pisou em falso e caiu. Ambas relataram que não apresentaram consequências físicas, mas possuem medo de cair novamente.

A ocorrência de quedas encontradas no presente estudo foi inferior aos achados em outros estudos da literatura, e no que se refere às consequências dessas quedas, a mais observada foi o medo de cair novamente que pode resultar em novos eventos de quedas, pois o medo resulta em insegurança por parte dos idosos em realizar atividades cotidianas. ${ }^{22}$

A partir do cenário exposto, da avaliação de saúde e dos riscos intrínsecos e extrínsecos para quedas, foi possível elencar as alterações em alguns domínios segundo a $\mathrm{CIPE}^{\circledR} / \mathrm{CIPESC}^{\circledR}$ sendo eles: nutrição, eliminação, exercícios e atividade física, mobilidade, percepção, ambiente, segurança e aprendizagem. Com isso, identificou-se os seguintes diagnósticos de enfermagem: emagrecimento, incontinência urinária, atividade física inadequada, andar comprometido, destreza manual diminuída, fraqueza, memória deficiente, risco para acidente doméstico - idoso, falta de conhecimento sobre prevenção de quedas e, risco de queda. ${ }^{35,36}$
A pertinência dos diagnósticos de enfermagem elencados para estratégias de cuidados em idosos da comunidade, estão de acordo com a literatura, uma vez que estudos demonstram a importância da utilização dos diagnósticos originados pela CIPE ${ }^{\circledR}$ para a identificação e prevenção precoce nos problemas identificados, resultando em maior resolutividade da assistência de enfermagem prestada as pessoas idosas. ${ }^{37,38}$ Para além disso, os diagnósticos elencados no presente estudo estão em consonância com um estudo metodológico realizado no estado do Ceará, que objetivou desenvolver uma nomenclatura de diagnósticos, resultados e intervenções de enfermagem para pessoas idosas acompanhadas na ESF, sendo que os diagnósticos utilizados pela CIPE $^{\circledR}$ favoreceram as especificações dos conceitos aperfeiçoando o processo de enfermagem. ${ }^{38}$

Ademais, um estudo realizado com 156 idosos da APS identificou que o diagnóstico risco de quedas esteve relacionado com os fatores de risco intrínsecos, como a idade $>65$ anos, presença de alterações cognitivas, deficiência visual, mobilidade e equilíbrio prejudicados, mas também com os fatores extrínsecos, relacionados principalmente com os riscos ambientais para quedas. Dessa forma, percebe-se que os diagnósticos elencados estão em consonância com a literatura, trazendo os principais fatores de riscos para quedas na população idosa que vive na comunidade. ${ }^{39}$

\section{Proposta de intervenção na realidade objetiva}

Nessa última etapa da TIPESC, foi proposto o plano de intervenções de enfermagem segundo a CIPE ${ }^{\circledR} /$ CIPESC $^{\circledR}$, balizada nos diagnósticos elencados na etapa anterior de captação da realidade, conforme descrito anteriormente (Quadro 1).

A escolha de realizar uma intervenção baseada na CIPE ${ }^{\circledR} /$ CIPESC $^{\circledR}$ se ancorou na importância da utilização de uma linguagem padronizada e de agregar evidência à prática da enfermagem sistematizando a assistência aos idosos do grupo de convivência pautada no conceito holístico, coerente com a abordagem de Pesquisa-Cuidado, uma vez que a CIPE $^{\circledR} /$ CIPESC $^{\circledR}$ possui características importantes de serem aplicadas na

Quadro 1. Diagnósticos e Intervenções de Enfermagem segundo a CIPE e CIPESC ${ }^{\circledR}$.

\begin{tabular}{|ll|}
\hline $\begin{array}{c}\text { Diagnóstico de } \\
\text { Enfermagem }\end{array}$ & \multicolumn{1}{c|}{ Intervenção de Enfermagem } \\
\hline Emagrecimento & $\begin{array}{l}\text { Avaliar as condições de moradia e ambiente familiar. Orientar sobre nutrição. Investigar hábitos } \\
\text { alimentares individuais e familiares. Monitorar peso corporal. Promover discussão sobre os } \\
\text { determinantes do emagrecimento. }\end{array}$ \\
$\begin{array}{ll}\text { Incontinência } \\
\text { urinária }\end{array}$ & $\begin{array}{l}\text { Agendar consulta médica na Unidade de Atenção à Saúde. Orientar sobre importância de higiene } \\
\text { íntima. Orientar sobre exercícios do períneo. }\end{array}$ \\
$\begin{array}{l}\text { Atividade física } \\
\text { inadequada }\end{array}$ & $\begin{array}{l}\text { Incentivar atividade física. Incentivar passeios e caminhadas. Orientar que ausência de exercício } \\
\text { (artrose), muscular e diminuição da mobilidade intestinal. Procurar caminhar em locais seguros e } \\
\text { apropriados. Promover discussão sobre os determinantes da ausência da realização de atividades } \\
\text { físicas. Realizar caminhadas e passeios. Tomar água antes, durante e depois da atividade física. }\end{array}$ \\
\hline
\end{tabular}

Fonte: as autoras, 2020. 
Quadro 1. Continuação...

\begin{tabular}{|c|c|}
\hline $\begin{array}{l}\text { Diagnóstico de } \\
\text { Enfermagem }\end{array}$ & Intervenção de Enfermagem \\
\hline $\begin{array}{l}\text { Andar } \\
\text { comprometido }\end{array}$ & $\begin{array}{l}\text { Esclarecer dúvidas sobre alterações na atividade motora. Identificar fatores desencadeantes da } \\
\text { atividade motora alterada. Usar calçados confortáveis e bem adaptados. }\end{array}$ \\
\hline $\begin{array}{l}\text { Destreza manual } \\
\text { diminuída }\end{array}$ & $\begin{array}{l}\text { Apoiar emocionalmente o paciente em suas necessidades. Avaliar capacidade física. Avaliar risco de } \\
\text { acidentes domésticos. Cuidar ao usar facas, tesouras, alicates etc. Cuidar quando mexer em objetos } \\
\text { aquecidos: fogão, panelas, ferro elétrico, lâmpadas. Envolver familiares para disponibilizar atividades } \\
\text { manuais ao Idoso. Estimular a participação em atividades físicas ou de lazer que envolvam a imagem } \\
\text { corporal. Fazer exercícios com bolinha de borracha, abrindo e fechando as mãos, três vezes ao dia, por } \\
\text { cinco minutos. Identificar situações de risco. Incentivar a realização de atividades manuais. }\end{array}$ \\
\hline Fraqueza & $\begin{array}{l}\text { Obter dados sobre atitude em relação à condição nutricional. Investigar origem da fraqueza. Agendar } \\
\text { consulta médica na Unidade Básica de Saúde. Orientar se levantar quando está se sentindo fraco. }\end{array}$ \\
\hline $\begin{array}{l}\text { Memória } \\
\text { deficiente }\end{array}$ & $\begin{array}{l}\text { Desenvolver atividades de reativação ou preservação da memória, por meio de jogos coloridos, } \\
\text { palavras cruzadas, bingos c/ números, figuras e cores. Estimular vínculo comunitário. Identificar } \\
\text { situações que dificultem o aprendizado. Oferecer apoio emocional. Orientar o idoso no tempo e no } \\
\text { espaço, mantendo um calendário e um relógio de fácil visualização. Participar de bingos. Verificar nível } \\
\text { de consciência. }\end{array}$ \\
\hline $\begin{array}{l}\text { Risco para acidente } \\
\text { doméstico - idoso }\end{array}$ & $\begin{array}{l}\text { Estar atento para piso escorregadio, retirando tapetes e/ou adaptando piso antiderrapante. Evitar } \\
\text { dirigir ou manusear equipamentos se estiver com tontura. Evitar mudanças bruscas de posição, } \\
\text { principalmente ao levantar-se da cama. Evitar tomar banho com a água superaquecida. Evitar } \\
\text { trancar a porta do banheiro durante o seu uso. Investigar riscos para acidentes domésticos. Não } \\
\text { se levantar no escuro. Orientar ambiente doméstico adequado. Providenciar barras de apoio onde } \\
\text { necessário. Providenciar tapetes emborrachados no banheiro/vaso sanitário e chuveiro. Usar calçados } \\
\text { confortáveis e bem adaptados. Utilizar assento para banho. }\end{array}$ \\
\hline $\begin{array}{l}\text { Falta de } \\
\text { conhecimento } \\
\text { sobre prevenção } \\
\text { de quedas }\end{array}$ & $\begin{array}{l}\text { Orientar sobre prevenção de quedas. Orientar família sobre prevenção de quedas. Promover } \\
\text { atividades nos Centros Comunitários de Saúde sobre prevenção de quedas. }\end{array}$ \\
\hline Risco de quedas & $\begin{array}{l}\text { Prevenir quedas. Orientar paciente/família sobre prevenção de quedas. Utilizar assento para banho. } \\
\text { Usar calçados confortáveis e bem adaptados. Evitar uso de tapetes soltos na casa. Evitar subir em } \\
\text { móveis. Orientar importância de iluminação suficiente em todos os ambientes. Fazer rastreamento do } \\
\text { risco de quedas. Providenciar barras de apoio onde necessário. }\end{array}$ \\
\hline
\end{tabular}

Fonte: as autoras, 2020.

APS e na prática do profissional enfermeiro. ${ }^{40}$ Assim, a partir dos diagnósticos e intervenções propostos, a enfermeira da ESF pode realizar o cuidado de enfermagem com foco na prevenção de quedas e agravos consequentes, favorecendo desse modo, o aumento da qualidade de vida dos idosos, e ressaltando que o cuidado de enfermagem inclui a interprofissionalidade.

A literatura aponta que as principais intervenções para o risco de quedas são a realização de exercícios físicos regulares que auxiliam na preservação da mobilidade dos idosos, retirada de medicamentos psicotrópicos por meio de avaliações e acompanhamento médico, avaliação e modificação de fatores de risco ambientais, corroborando assim com as intervenções propostas no presente estudo. ${ }^{41,42}$

Ainda, é imprescindível ressaltar que a problemática das quedas vem sendo abordada com as idosas participantes do grupo "De bem com a vida" ao longo dos anos por meio de diversas atividades e projetos que são realizados com elas, uma vez que a adesão delas é significativa. Dessa forma, a partir da elaboração da proposta de intervenção na realidade objetiva, realizou-se pactuação com a enfermeira da unidade investigada através do diálogo por meio de reuniões, para que pudesse, juntamente com a enfermeira e demais membros da equipe, implantar as intervenções junto às idosas.

\section{CONCLUSÕES E IMPLICAÇÕES PARA PRÁTICA}

A partir do estudo foi possível analisar o processo de cuidado para prevenção de quedas em idosos por meio da perspectiva teórica e metodológica da Teoria de Intervenção Práxica da 
Enfermagem em Saúde Coletiva. Ao realizar a captação e interpretação da realidade objetiva observou-se que existem estratégias para a prevenção de quedas na APS e que as idosas participantes do grupo de convivência possuem riscos de quedas relacionados aos fatores intrínsecos e extrínsecos.

Portanto, a partir da avaliação do risco de quedas das idosas foi traçado um plano de intervenções, elencando os principais diagnósticos e intervenções de enfermagem segundo a CIPE $^{\circledR}$ e a CIPESC ${ }^{\circledR}$, relacionando aos domínios afetados de nutrição, eliminação, exercícios e atividade física, mobilidade, percepção, ambiente, segurança e aprendizagem. Assim, o estudo contribuiu para preencher a lacuna do conhecimento sobre os riscos de quedas mais frequentes em idosas participantes de um grupo de convivência no âmbito da APS, considerando a importância dela para a saúde do idoso.

Como limitações do estudo, ressalta-se que devido a coleta de dados ocorrer durante o período de pandemia de COVID-19, não foi possível que as entrevistas fossem realizadas presencialmente, uma vez que a população-alvo do estudo é vulnerável para essa doença, tendo também suas Atividades de Vida Diária alteradas. Por isso, não houve a aplicação na íntegra do questionário MEEM, que foi realizada via telefone, e tampouco houve a avaliação presencial dos riscos ambientais do domicílio dessas idosas. Recomenda-se que sejam feitos novos estudos sobre os fatores de riscos de quedas em idosos, permitindo analisar como o grupo de convivência interfere nesses fatores e em outras realidades, bem como a realização de todas as etapas da TIPESC, avaliando assim a efetividade do plano de intervenção proposto.

\section{CONTRIBUIÇÕES DOS AUTORES}

Desenho do estudo. Larissa Padoin Lopes. Iara Sescon Nogueira. Jhenicy Rubira Dias. Vanessa Denardi Antoniassi Baldissera

Aquisição de dados. Larissa Padoin Lopes. Iara Sescon Nogueira. Jhenicy Rubira Dias

Análise de dados e interpretação dos resultados. Larissa Padoin Lopes. Iara Sescon Nogueira. Jhenicy Rubira Dias. Vanessa Denardi Antoniassi Baldissera

Redação e revisão crítica do manuscrito. Larissa Padoin Lopes. Iara Sescon Nogueira. Jhenicy Rubira Dias. Vanessa Denardi Antoniassi Baldissera

Aprovação da versão final do artigo. Larissa Padoin Lopes. lara Sescon Nogueira. Jhenicy Rubira Dias. Vanessa Denardi Antoniassi Baldissera

Responsabilidade por todos os aspectos do conteúdo e a integridade do artigo publicado. Larissa Padoin Lopes. lara Sescon Nogueira. Jhenicy Rubira Dias. Vanessa Denardi Antoniassi Baldissera

\section{EDITOR ASSOCIADO}

Cristina Lavareda Baixinho (1)

\section{EDITOR CIENTÍFICO}

\author{
Marcelle Miranda da Silva (1)
}

\section{REFERÊNCIAS}

1. Pena SB, Guimarães HCQCP, Lopes JL, Guandalini LS, Taminato M, Barbosa DA et al. Medo de cair e o risco de queda: revisão sistemática e metanálise. Acta Paul Enferm. 2019 ago;32(4):456-63. http://dx.doi. org/10.1590/1982-0194201900062.

2. Smith AA, Silva AO, Rodrigues RAP, Moreira MASP, Nogueira JA, Tura LFR. Assessment of risk of falls in elderly living at home. Rev LatinoAm Enfermagem. 2017;25:e2754. http://dx.doi.org/10.1590/15188345.0671.2754. PMid:28403333.

3. Chini LT, Pereira DS, Nunes AA. Validação da Ferramenta de Rastreio de Risco de quedas (FRRISque) em pessoas idosas que vivem na comunidade. Cien Saude Colet. 2019;24(8):2845-58. http://dx.doi. org/10.1590/1413-81232018248.28962017. PMid:31389533.

4. Ang GC, Low SL, How $\mathrm{CH}$. Approach to falls among the elderly in the community. Singapore Med J. 2020;61(3):116-21. http://dx.doi. org/10.11622/smedj.2020029. PMid:32488276.

5. Silveira MB, Saldanha RP, Leite JCC, Silva TOFD, Silva T, Filippin LI Construction and validation of content of one instrument to assess falls in the elderly. Einstein (Sao Paulo). 2018 jun 11;16(2):eAO4154. http:// dx.doi.org/10.1590/s1679-45082018ao4154. PMid:29898025.

6. Souza AMG, Ferreira TLS, Santos KMR, Oliveira DJD, Andrade FB Avaliação da assistência à pessoa idosa na atenção primária à saúde: perspectiva de usuários. Rev Ciênc Plur. 2017;3(2):42-52. http://dx.doi. org/10.21680/2446-7286.2017v3n2ID12705.

7. Araújo LSA, Moreira ACA, Freitas CASL, Silva MAM, Val DA. Idosos e grupos de convivência: motivos para não adesão. SANARE. [Internet] 2017 [citado 2021 jun 9];16(1):58-67. Disponível em: https://sanare. emnuvens.com.br/sanare/article/view/1140

8. Guerra HS, Bernardes DCF, Santana JÁ, Barreira LM, Sousa RA, Neves CM. Avaliação do risco de quedas em idosos da comunidade. Revista Saúde Com. 2017;13(2):879-86. http://dx.doi.org/10.22481/ rsc.v13i2.434.

9. Egry EY, Fonseca RMGS, Oliveira MAC, Bertolozzi MR. Nursing in Collective Health: reinterpretation of objective reality by the praxis action. Rev Bras Enferm. 2018;71(Suppl 1):758-63. http://dx.doi. org/10.1590/0034-7167-2017-0677.

10. Lacerda MR, Giacomozzi CM, Przenyczka RA, Camargo TB. Pesquisaação, pesquisa convergente assistencial e pesquisa cuidado no contexto da enfermagem: semelhanças e peculiaridades. Rev Eletr Enferm 2008;10(3):843-8. http://dx.doi.org/10.5216/ree.v10.46705.

11. Ministério da Saúde (BR). Departamento de Atenção Básica. Envelhecimento da pessoa idosa. Brasília: Ministério da Saúde; 2006. Caderno da atenção básica; 19.

12. Lawton MP, Brody EM. Assessment o folder people: self-maintaining and instrumental activities of daily living. Gerontologist. 1969;9(3):17986. http://dx.doi.org/10.1093/geront/9.3_Part_1.179. PMid:5349366.

13. Moraes EM, Moraes FL. Avaliação multidimensional do idoso. $5^{\underline{a}}$ ed Belo Horizonte (MG): Folium; 2016.

14. Secretaria de Estado da Saúde do Paraná (PR). Superintendência de Atenção à Saúde. Linha guia da saúde do idoso. Curitiba: SESA;2017. $149 \mathrm{p}$.

15. Secretaria de Estado da Saúde do Paraná (PR). Plano Estadual de Saúde Paraná 2016-2019. Curitiba: SESA; 2016. 200 p.

16. Prefeitura Municipal de Maringá (PR). Secretaria Municipal de Saúde de Maringá. Assessoria de Planejamento. Plano Municipal de Saúde de Maringá-PR, 2018-2021 [Internet]. Maringá: Prefeitura Municipal; 2018 [citado 2021 jun 9]. Disponível em: http://www2.maringa.pr.gov. br

17. Ferreira MCG, Tura LFR, Silva RCD, Ferreira MA. Social representations of older adults regarding quality of life. Rev Bras Enferm. 2017 jul/ ago;70(4):806-13. http://dx.doi.org/10.1590/0034-7167-2017-0097. PMid:28793112. 
18. Kim T, Choi SD, Xiong S. Epidemioloy of fall and its socioeconomic risk factors in community-dwelling Korean elderly. PLoS One. 2020;15(6):e0234787. http://dx.doi.org/10.1371/journal.pone.0234787. PMid:32559206.

19. Silva FA, Matos MIB, Esteves LSF. Avaliação do risco de quedas em idosos independentes. Colloq Vitae. 2017;9(1):18-22. http://dx.doi. org/10.5747/cv.2017.v09.n1.v184

20. Lima AP, Lini EV, Dellani MP, Portella MR, Doring M. Prevalência e fatores associados às quedas em idosos de Estação-RS: estudo transversal de base populacional. Cad Saúde Coletiva. 2017;25(4):436-42. http:// dx.doi.org/10.1590/1414-462x201700040271.

21. Santos EC, Couto BM, Bastone $A C$. Fatores associados à autoavaliação negativa da saúde em idosos cadastrados nas Unidades Básicas de Saúde. ABCS Health Sci. 2018;43(1):47-57. http://dx.doi.org/10.7322/ abcshs.v43i1.999.

22. De Sousa-Araújo IV, C Gomes N, Santos-Nascimento J, Neves Romanato Ribeiro CC, Dos Santos Tavares DM. Quedas entre idosos: preditores e distribuição espacial. Rev Salud Publica (Bogota). 2019;21(2):187-94. http://dx.doi.org/10.15446/rsap.v21n2.70298. PMid:33027328.

23. Souza LHR, Brandão JCS, Fernandes AKC, Cardoso BLC. Quedas em idosos e fatores de risco associados. Rev Aten Saúde. 2017;15(54):5560. http://dx.doi.org/10.13037/ras.vol15n54.4804.

24. Costa-Dias MJ, Oliveira AS, Martins T, Araújo F, Santos AS, Moreira $\mathrm{CN}$, et al. Medication fall risk in old hospitalized patients: a retrospective study. Enfermeira Educ hoje. 2014;34(2):171-6. http://dx.doi.org/10.1016/j. nedt.2013.05.016.

25. Ming Y, Zecevic A. Medications \& polypharmacy influence on recurrent fallers in community: a systematic review. Can Geriatr J.2018;21(1):14 25. http://dx.doi.org/10.5770/cgj.21.268. PMid:29581817.

26. Marinho CL, Nascimento V, Bonadiman BSR, Torres SRF. Causas consequências de quedas de idoso em domicílio. Braz. J. Hea. Rev. 2020;3(3):6880-96. http://dx.doi.org/10.34119/bjhrv3n3-225.

27. Miranda DP, Santos TD, Santo FEE, Chibante CLP, Barreto EA. Quedas em idosos em ambiente domiciliar: uma revisão integrativa. REAID. [Internet]. 2017; [citado 2021 jun 9];120-9. Disponível em: https://www. revistaenfermagematual.com.br/index.php/revista/article/view/560

28. Oliveira CES, Felipe SGB, Silva CRDT, Carvalho DB, Silva-Júnior F, Figueiredo MLF et al. Vulnerabilidade clínico-funcional de idosos em um centro de convivência. Acta Paul Enferm. 2020;33:1-8. http://dx.doi. org/10.37689/acta-ape/2020AO0172.

29. Queiroz ACCN, Feitosa COPS, Rodrigues GMM, Sousa JC. Intervenções na prevenção de quedas de idosos em ambiente domiciliar. ReBIS [Internet]. 2020 [citado 2021 jun 9];2(4):1-5. Disponível em: https:// revistarebis.rebis.com.br/index.php/rebis/article/view/115

30. Gonçalves ERS, Gaspar ACM, Vechia ADRD, Azevedo RCS, Reiners AAO. Environmental risk factors, prevalence and consequences of falls in the elderly's home. Rev Enferm UFPI. 2020;9:e10458. http://dx.doi. org/10.26694/reufpi.v9i0.10458.

31. Teixeira DKS, Andrade LM, Santos JLP, Caires ES. Quedas em pessoas idosas: restrições do ambiente doméstico e perdas funcionais. Rev Bras Geriatr Gerontol. 2019;22(3):1-10. http://dx.doi.org/10.1590/198122562019022.180229.
32. Borges PS, Marinho Fo LEN, Mascarenhas CHM. Correlação entre equilíbrio e ambiente domiciliar como risco de quedas em idosos com acidente vascular encefálico. Rev Bras Geriatr Gerontol. 2019;13(1):4550. http://dx.doi.org/10.1590/S1809-98232010000100005.

33. Rodrigues GD, Barbieiro AB, Junior EDA. Prevenção de quedas no idoso: revisão de literatura brasileira. Rev Bras Prescr Fisiol Exerc. [Internet]. 2016 [citado 2021 jun 9];10(59):431-7. Disponível em: https:// dialnet.unirioja.es/servlet/articulo?codigo $=5587489$

34. Ferreira LMBM, Jerez-Roig J, Andrade FLJP, Oliveira NPD, Araújo JRT, Lima KC. Prevalence of falls and evaluation of mobility among institutionalized elderly persons. Rev Bras Geriatr Gerontol. 2016 dez;19(6):995-1003. http://dx.doi.org/10.1590/1981-22562016019.160034.

35. Conselho Internacional de Enfermeiros. Enfermagem Comunitária: Um projeto partilhado com o governo escocês e o Serviço Nacional de Saúde da Escócia. Catálogo da Classificação Internacional para a Prática de Enfermagem (CIPE $®)$ [Internet]. Lisboa, Portugal: Ordem dos Enfermeiros; 2015 [citado 2021 jun 9]. 56 p. Disponível em: https://www.ordemenfermeiros.pt/arquivo/publicacoes/Documents/ CatalogoCIPE web julho2015.pdf

36. Albuquerque LM, Cubas MR, organizadores. Classificação Internaciona das Práticas de Enfermagem em Saúde Coletiva [Internet]. São Paulo: Prefeitura da Cidade de São Paulo; 2005. Consulta de Diagnósticos de Enfermagem. Extraído do livro CIPESCANDO EM CURITIBA: Construção e implementação da Nomenclatura de Diagnósticos e intervenções de enfermagem na Rede Básica de Saúde [citado 2021 jul 1]. 65 p. Disponível em: https://www.ribeiraopreto.sp.gov.br/files/ ssaude/pdf/cipesc.pdf

37. Andrada MMC, França M, Alvarez AM, Hammerschmid KSA. Diagnósticos de enfermagem para idosos internados, segundo a Classificação Internacional das Práticas de Enfermagem. Rev Rene. 2015;16(1):97105. http://dx.doi.org/10.15253/2175-6783.2015000100013.

38. Clares JWB, Nóbrega MML, Guedes MVC, Silva LF, Freitas MC. ICNP® nursing diagnoses, outcomes and interventions for community elderly. Rev Bras Enferm. 2019;72(Suppl 2):191-8. http://dx.doi.org/10.1590/00347167-2018-0540. PMid:31826210.

39. Santos PHFD, Stival MM, Lima LR, Santos WS, Volpe CRG, Rehem TCMSB et al. Nursing diagnosis Risk for Falls in the elderly in primary healthcare. Rev Bras Enferm. 2020;73(73, Suppl 3):e20180826. http:// dx.doi.org/10.1590/0034-7167-2018-0826. PMid:32667409.

40. Crivelaro PMD, Fidelis FAM, Siviero MRS, Borges PFB, Gouvêa AHM Papini SJ. O processo de enfermagem e classificação internacional para a prática de enfermagem (CIPE®): Potencialidades na atenção primária. Braz J of Dev. 2020;6(7):54085-101. http://dx.doi.org/10.34117/ bjdv6n7-889.

41. Tricco AC, Thomas SM, Veroniki AA, Hamid JS, Cogo E, Strifler AC et al. Comparisons of interventions for preventing falls in older adults: a systematic review and meta-analysis. JAMA. 2017;318(17):1687-99. http://dx.doi.org/10.1001/jama.2017.15006. PMid:29114830.

42. Cheng P, Tan L, Ning P, Li L, Gao Y, WuY et al. Comparative effectiveness of published interventions for elderly fall prevention: a systematic review and network meta-analysis. Int J Environ Res Public Health. 2018;15(3):114. http://dx.doi.org/10.3390/ijerph15030498. PMid:29534531. 